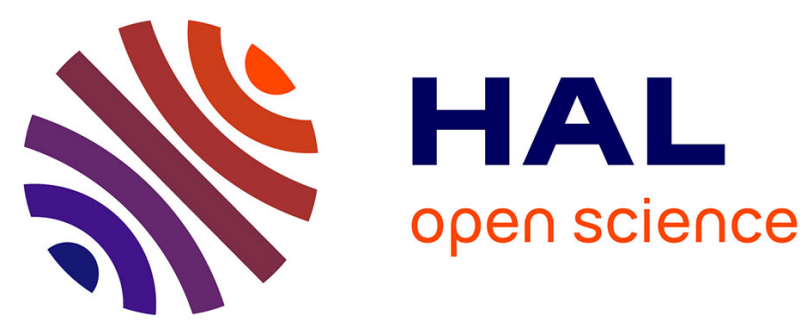

\title{
Benzonitrile as a Proxy for Benzene in the Cold ISM: Low-temperature Rate Coefficients for CN + C 6 H 6
}

Ilsa R. Cooke, Divita Gupta, Joseph Messinger, Ian R Sims

\section{To cite this version:}

Ilsa R. Cooke, Divita Gupta, Joseph Messinger, Ian R Sims. Benzonitrile as a Proxy for Benzene in the Cold ISM: Low-temperature Rate Coefficients for CN + C $6 \mathrm{H} 6$. The Astrophysical journal letters, 2020, 891 (2), pp.L41. 10.3847/2041-8213/ab7a9c . hal-02510885

HAL Id: hal-02510885

https://hal-univ-rennes1.archives-ouvertes.fr/hal-02510885

Submitted on 18 Mar 2020

HAL is a multi-disciplinary open access archive for the deposit and dissemination of scientific research documents, whether they are published or not. The documents may come from teaching and research institutions in France or abroad, or from public or private research centers.
L'archive ouverte pluridisciplinaire HAL, est destinée au dépôt et à la diffusion de documents scientifiques de niveau recherche, publiés ou non, émanant des établissements d'enseignement et de recherche français ou étrangers, des laboratoires publics ou privés. 


\title{
Benzonitrile as a Proxy for Benzene in the Cold ISM: Low-temperature Rate Coefficients for $\mathbf{C N}+\mathbf{C}_{6} \mathbf{H}_{6}$
}

\author{
Ilsa R. Cooke ${ }^{1}(\mathbb{D})$, Divita Gupta ${ }^{1}\left(\mathbb{D}\right.$, Joseph P. Messinger ${ }^{1,2}$ (D), and Ian R. Sims ${ }^{1}$ (D) \\ ${ }^{1}$ Univ Rennes, CNRS, IPR (Institut de Physique de Rennes)_UMR 6251, F-35000 Rennes, France; ian.sims@univ-rennes1.fr \\ 2 Division of Chemistry and Chemical Engineering, California Institute of Technology, Pasadena, CA 91125, USA \\ Received 2020 January 23; revised 2020 February 27; accepted 2020 February 27; published 2020 March 16
}

\begin{abstract}
The low-temperature reaction between $\mathrm{CN}$ and benzene $\left(\mathrm{C}_{6} \mathrm{H}_{6}\right)$ is of significant interest in the astrochemical community due to the recent detection of benzonitrile, the first aromatic molecule identified in the interstellar medium (ISM) using radio astronomy. Benzonitrile is suggested to be a low-temperature proxy for benzene, one of the simplest aromatic molecules, which may be a precursor to polycyclic aromatic hydrocarbons. In order to assess the robustness of benzonitrile as a proxy for benzene, low-temperature kinetics measurements are required to confirm whether the reaction remains rapid at the low gas temperatures found in cold dense clouds. Here, we study the $\mathrm{C}_{6} \mathrm{H}_{6}+\mathrm{CN}$ reaction in the temperature range 15-295 K, using the well-established CRESU technique (a French acronym standing for Reaction Kinetics in Uniform Supersonic Flow) combined with pulsed-laser photolysis-laser-induced fluorescence. We obtain rate coefficients, $k(T)$, in the range $(3.6-5.4) \times 10^{-10} \mathrm{~cm}^{3} \mathrm{~s}^{-1}$ with no obvious temperature dependence between 15 and $295 \mathrm{~K}$, confirming that the $\mathrm{CN}+\mathrm{C}_{6} \mathrm{H}_{6}$ reaction remains rapid at temperatures relevant to the cold ISM.
\end{abstract}

Unified Astronomy Thesaurus concepts: Reaction rates (2081); Astrochemistry (75); Interstellar molecules (849); Interstellar medium (847); Interdisciplinary astronomy (804); Polycyclic aromatic hydrocarbons (1280); Dense interstellar clouds (371); Interstellar clouds (834)

\section{Introduction}

The recent discovery of benzonitrile $\left(\mathrm{C}_{6} \mathrm{H}_{5} \mathrm{CN}\right)$ in a nearby cold molecular cloud (Taurus, TMC-1) marks the first detection of an aromatic species in the interstellar medium by radio astronomy (McGuire et al. 2018). Benzonitrile has been proposed as a tracer of benzene, which may be a lowtemperature precursor to more complex polycyclic aromatic hydrocarbons (PAHs). PAHs are widely accepted to exist in the interstellar medium (ISM) owing to their characteristic infrared emission features; however, due to their structural similarities, the exact chemical origins of the IR bands remain elusive (Lovas et al. 2005). It has been suggested that PAHs make up as much as $10 \%-25 \%$ of the interstellar carbon budget (Dwek et al. 1997; Chiar et al. 2013). Understanding the origin of PAHs can help answer fundamental questions about their role in forming interstellar dust as well as potentially prebiotic material that may be incorporated into new planetary systems.

It has been suggested that a bottleneck to the formation of these large aromatics at low temperatures is the cyclization to produce the first aromatic ring, usually benzene or the phenyl radical (Cherchneff et al. 1992; Tielens \& Charnley 1997; Kaiser et al. 2015). However, benzene itself is difficult to detect as it has no permanent dipole moment and hence is invisible to radio astronomy. While benzene has been detected in the ISM through infrared observations of a single weak absorption feature (the $\nu_{4}$ bending mode near $14.85 \mu \mathrm{m}$ ) in a handful of sources (Cernicharo et al. 2001; Kraemer et al. 2006; Malek et al. 2011), absorption due to Earth's atmosphere limits its observation to bright IR-sources using space-based infrared telescopes (e.g., the Spitzer Space Telescope and the Infrared Space Observatory). Instead, it was suggested that benzonitrile (dipole moment $=4.5 \mathrm{D}$ ) might be used as a chemical proxy for benzene in the cold, starless ISM, as it is expected to form via the barrierless, exothermic neutral-neutral reaction between
CN and benzene (Woods et al. 2002; Trevitt et al. 2010). If linked to benzene, benzonitrile may be used to constrain the early stages of the aromatic chemistry in the ISM.

Following a tentative detection using data from the Nobeyama $45 \mathrm{~m}$ telescope, McGuire et al. (2018) conducted a deep integration search for benzonitrile in TMC-1 with the $100 \mathrm{~m}$ Robert C. Byrd Green Bank Telescope. They observed eight rotational transitions between 18 and $23 \mathrm{GHz}$, several of which displayed resolved hyperfine splitting, confirming the detection of benzonitrile and allowing derivation of its column density, $N_{T}=4 \times 10^{11} \mathrm{~cm}^{-2}$.

McGuire et al. (2018) modified the kida.uva.2014 reaction network (Wakelam et al. 2015) to include the $\mathrm{CN}+\mathrm{C}_{6} \mathrm{H}_{6}$ reaction, as well as a handful of destruction pathways in order to predict the abundance of benzonitrile in TMC-1. Currently, the KIDA database ${ }^{3}$ does not include benzonitrile but does include 13 ion-neutral pathways for benzene destruction and only five neutral-neutral pathways (mostly $\mathrm{H}$-abstraction mechanisms). McGuire et al. (2018) used a reaction rate coefficient for $\mathrm{CN}+\mathrm{C}_{6} \mathrm{H}_{6}$ of $3 \times 10^{-10} \mathrm{~cm}^{3} \mathrm{~s}^{-1}$, based on the assumption that the reaction will occur upon every collision. Their model predicted a benzonitrile abundance a factor of four lower than what was observed in TMC-1. The authors suggested that the difference may be explained by other formation routes for benzene and/or benzonitrile that were not considered in their model.

Rate coefficients for the reaction between $\mathrm{CN}$ and benzene have been previously measured using Pulsed-Laser PhotolysisLaser Induced Fluorescence (PLP-LIF) at 295, 165, and $105 \mathrm{~K}$ by Trevitt et al. (2010), who found $k(T)$ to be relatively constant, ranging from 3.9 to $4.9 \times 10^{-10} \mathrm{~cm}^{3} \mathrm{~s}^{-1}$. They attribute the lack of temperature dependence to a reaction mechanism that proceeds without an energy barrier in the

\footnotetext{
http://kida.astrophy.u-bordeaux.fr/, accessed 2020 January.
} 
entrance channel and likely forms an addition complex, which is supported by theoretical calculations of the reaction potential energy surface (Balucani et al. 1999; Woon 2006; Lee et al. 2019). In addition to the overall rate coefficients, Trevitt et al. (2010) measured the products of the reaction at room temperature using synchrotron VUV photoionization mass spectrometry. They found that the photoionization efficiency curve at $m / z=103$ could be well fit by benzonitrile photoionization spectrum with no detectable evidence for the $\mathrm{H}$-abstraction product channel, $\mathrm{C}_{6} \mathrm{H}_{5}+\mathrm{HCN}$, nor the $-\mathrm{NC}$ isomer phenylisocyanide.

Here, we report rate coefficients for the reaction of benzene with the $\mathrm{CN}$ radical over a wide temperature range of 15-295 K using the Cinétique de Reaction en Ecoulement Supersonique Uniforme (or Reaction Kinetics in Uniform Supersonic Flow; CRESU) technique. These rate coefficients can be input into astrochemical models to assess the importance of the reaction as a production route for benzonitrile and therefore the robustness of benzonitrile as a chemical proxy for benzene at various temperatures in the ISM.

\section{Experiment}

The kinetics of the reaction between the $\mathrm{CN}$ radical and benzene were measured using PLP-LIF. The low gas temperatures were achieved using the CRESU technique, which has been described in detail previously (Sims et al. 1994; James et al. 1998; Cooke \& Sims 2019) and will only be described here in brief. A dilute mixture (typically $<0.1 \%$ ) of benzene (Sigma Aldrich, Anhydrous 99.8\%) and the $\mathrm{CN}$ radical precursor in a buffer gas (He (99.995\%), Ar (99.998\%), or $\mathrm{N}_{2}$ (99.995\%); Air Liquide) expands isentropically from a high-pressure region (reservoir), into a low-pressure region (chamber) through a convergent-divergent Laval nozzle to generate a cold supersonic flow that is uniform in temperature and density for several tens of centimeters, corresponding typically to $100-500 \mu \mathrm{s}$. The high molecular density of the flow $\left(10^{16}-10^{17} \mathrm{~cm}^{-3}\right)$ results in collisions between the molecules, ensuring thermal equilibrium is maintained. A wide range of temperatures can be obtained by careful manipulation of the physical dimensions of the nozzle, the buffer gas used, and the flow conditions/pumping capacity. Characterizing the flow temperature is essential to study chemical kinetics and is determined using Pitot probe impact pressure measurements for each nozzle prior to undertaking kinetics experiments.

A Controlled Evaporation and Mixing (CEM) system was used to introduce benzene into the flow (Bronkhorst CEM; described in detail in Gupta et al. 2019). The system consists of a Coriolis liquid flow meter (Bronkhorst mini CORI-FLOW ML120V00) that introduces a metered flow of liquid benzene into an evaporator (Bronkhorst W-202A-222-K), followed by controlled dilution with a buffer gas via a thermal mass flow controller (Bronkhorst EL-FLOW Prestige). The $\mathrm{CN}$ radicals were generated by photodissociation of ICN (Acros Organics, $98 \%$ ) at $248 \mathrm{~nm}$ using a $\mathrm{KrF}$ excimer laser (Coherent LPXPro 210) at a laser fluence in the reaction zone of $\sim 25 \mathrm{~mJ} \mathrm{~cm}^{-2}$. Photodissociation of ICN at $248 \mathrm{~nm}$ produces $\mathrm{CN}$ radicals primarily $(\geqslant 93 \%)$ in the $v=0$ level of the $X^{2} \Sigma^{+}$state (O'Halloran et al. 1987), and rapid rotational relaxation is ensured by frequent collisions due to the high density of the buffer gas.

The LIF/fluorescence signal was recorded with a photomultiplier tube (PMT; Thorn E6723) by excitation in the $(0,0)$ band of the $B^{2} \Sigma^{+}-X^{2} \Sigma^{+}$electronic transition at $\sim 388 \mathrm{~nm}$ using a dye laser (Laser Analytical Systems, LDL 20505, operating with $0.2 \mathrm{~g} \mathrm{l}^{-1}$ mixture of Exciton Exalite 389 dye in 1,4dioxane) pumped by the frequency-tripled output at $355 \mathrm{~nm}$ of a Nd:YAG laser (Continuum, Powerlite Precision II). A narrowband interference filter centered at $420 \mathrm{~nm}$ (Ealing Optics, $10 \mathrm{~nm}$ FWHM) was used to select the off-resonant fluorescence into the first vibrational level of the ground state via the $(0,1)$ band. Delays ranging from -5 to hundreds of microseconds between the excimer and the LIF dye laser pulses, both operating at $10 \mathrm{~Hz}$, were employed to record the time dependence of the $\mathrm{CN}$ radical fluorescence during the reaction.

The experiments were performed under pseudo-first-order conditions with benzene in excess. The UV absorption crosssection of ICN at $248 \mathrm{~nm}$ has been measured as $4.7 \times 10^{-19}$ $\mathrm{cm}^{2}$ (Felps et al. 1991). For the density of ICN used in the gas flow $\left(\sim 10^{12} \mathrm{~cm}^{-3}\right)$, there is an estimated $\mathrm{CN}\left(X^{2} \Sigma^{+}\right)$ concentration of $<1 \times 10^{10} \mathrm{~cm}^{-3}$, which is lower than the concentration of benzene by at least a factor of one hundred.

The LIF signals were recorded using a gated integrator (Stanford Research Systems) at 400 evenly spaced time delays and were averaged typically 5 times. These were fit to single exponential decays using Scipy's curve fit optimization package (Virtanen et al. 2019), yielding pseudo-first-order rate coefficients, $k_{1 \mathrm{st}}$. Plots of $k_{1 \mathrm{st}}$ versus the benzene concentration were the fit with a weighted linear regression (Seabold et al. 2017, as developed on GitHub) to calculate the second-order rate coefficient. The procedure was repeated for various temperatures using eight different Laval nozzles. The experimental parameters and the measured rate coefficients with their uncertainties are summarized in Table 1.

\section{Results}

Figure 1(a) shows an example CN LIF decay trace and second-order kinetics plot, obtained at $72 \mathrm{~K}$. The signal decays were recorded for $140-200 \mu$ s following the firing of the photolysis laser to capture the fast $\mathrm{CN}$ decay in the presence of benzene. LIF measurements were also taken at negative time delays ( $5 \mu$ s before the excimer laser fires) to establish a pretrigger baseline. An exponential decay function was fit to the LIF data after $\geqslant 10 \mu$ s to allow enough time for rotational relaxation of $\mathrm{CN}$ and for the photomultiplier tube to recover from scatter due to the excimer laser.

The LIF decay traces were recorded for the $\mathrm{CN}$ decay in the presence of at least eight different concentrations of benzene. The number of points and the range of benzene concentration used for each nozzle are shown in Table 1. The upper limit for the concentration of benzene is determined by an experimental constraint imposed due to the formation of benzene dimers, which could cause the rate coefficients to be underestimated. This effect is particularly significant at low temperatures, where dimerization occurs at much lower reactant concentrations than at room temperature. Hamon et al. (2000) measured the rate coefficients for dimerization of benzene in helium. They found that the onset of significant complex formation at $25 \mathrm{~K}$ occurred when $\left[\mathrm{C}_{6} \mathrm{H}_{6}\right] \geqslant 1 \times 10^{14} \mathrm{~cm}^{-3}$. We obtain the bimolecular rate coefficients by fitting benzene concentrations $<2.5 \times 10^{13} \mathrm{~cm}^{-3}$, except for at room temperature, where the fit concentrations are $<1 \times 10^{14} \mathrm{~cm}^{-3}$.

The nonzero intercept observed in the second-order plots (e.g., in Figure 1(b)) is due to other losses of $\mathrm{CN}$, a 
Table 1

Rate Coefficients for the Reaction of the CN Radical with Benzene Measured at Different Temperatures, with the Associated Experimental Parameters

\begin{tabular}{|c|c|c|c|c|c|}
\hline $\begin{array}{l}T \\
(\mathrm{~K})\end{array}$ & Buffer Gas & $\begin{array}{l}\text { Total Density } \\
\left(10^{16} \mathrm{~cm}^{-3}\right)\end{array}$ & $\begin{array}{l}\text { Range of }\left[\mathrm{C}_{6} \mathrm{H}_{6}\right] \\
\quad\left(10^{12} \mathrm{~cm}^{-3}\right)\end{array}$ & No. of Points & $\begin{array}{l}\text { Rate Coefficient, } k(T) \\
\qquad\left(10^{-10} \mathrm{~cm}^{3} \mathrm{~s}^{-1}\right)\end{array}$ \\
\hline 15 & $\mathrm{He}$ & 5.02 & $\begin{array}{l}2.19-19.7 \\
2.19-17.5\end{array}$ & $\begin{array}{l}13 \\
14\end{array}$ & $\begin{array}{c}5.45 \pm 0.90 \\
5.36 \pm 0.86 \\
\mathbf{5 . 4} \pm \mathbf{0 . 6}\end{array}$ \\
\hline 17 & $\mathrm{He}$ & 4.85 & $2.03-14.2$ & 13 & $4.5 \pm 0.7$ \\
\hline 72 & $\mathrm{He}$ & $\begin{array}{l}6.01 \\
6.01\end{array}$ & $\begin{array}{l}1.54-24.6 \\
3.00-16.5\end{array}$ & $\begin{array}{l}14 \\
12\end{array}$ & $\begin{aligned} 5.47 & \pm 0.63 \\
5.12 & \pm 0.73 \\
\mathbf{5 . 4} & \pm \mathbf{0 . 6}\end{aligned}$ \\
\hline 110 & $\mathrm{Ar}$ & 2.71 & $1.66-19.9$ & 11 & $4.2 \pm 0.6$ \\
\hline 200 & $\mathrm{~N}_{2}$ & 5.27 & $2.52-20.2$ & 13 & $3.7 \pm 0.8$ \\
\hline 294 & $\mathrm{~N}_{2}$ & 7.04 & $1.52-76.1$ & 9 & $3.47 \pm 0.40$ \\
\hline 293 & $\begin{array}{l}\mathrm{N}_{2} \\
\mathrm{~N}_{2}\end{array}$ & $\begin{array}{l}9.78 \\
18.5\end{array}$ & $\begin{array}{l}1.53-76.6 \\
2.21-69.6\end{array}$ & $\begin{array}{l}9 \\
8\end{array}$ & $\begin{array}{l}3.45 \pm 0.39 \\
3.33 \pm 0.54\end{array}$ \\
\hline 293 & $\begin{array}{l}\mathrm{He} \\
\mathrm{He} \\
\mathrm{He}\end{array}$ & $\begin{array}{l}5.27 \\
6.92 \\
9.39\end{array}$ & $\begin{array}{l}6.25-37.5 \\
2.03-81.3 \\
2.16-63.4\end{array}$ & $\begin{array}{c}9 \\
10 \\
6\end{array}$ & $\begin{array}{l}3.98 \pm 0.57 \\
3.35 \pm 0.46 \\
4.08 \pm 0.55\end{array}$ \\
\hline 295 & $\mathrm{He}$ & 9.10 & $1.50-74.8$ & 9 & $\begin{aligned} 4.02 & \pm 0.46 \\
\mathbf{3 . 6} & \pm \mathbf{0 . 4}\end{aligned}$ \\
\hline
\end{tabular}

Note. Quoted uncertainties are calculated using the standard error evaluated from the second-order plot, multiplied by the appropriate Student's $t$ factor for $95 \%$ confidence, and then combined in quadrature with an estimated systematic error of $10 \%$. Entries in bold are the variance weighted mean values of rate coefficients measured at the same temperature, where the standard error on the mean is combined in quadrature with an estimated systematic error of $10 \%$.

combination of $\mathrm{CN}$ diffusion out of the probed beam area and reaction with ICN and/or other impurities in the buffer gas. The second-order rate coefficients are derived from the slopes of the weighted linear least-squares regressions to the secondorder kinetics plots and are shown in Table 1 along with their uncertainties, which are calculated using $95 \%$ confidence limits of the standard error from the two-sided Student's $t$ distribution combined with an estimated $10 \%$ systematic error. The measured rate coefficients over the temperature range $T=15-295 \mathrm{~K}$ are shown in Figure 2 along with the rate coefficients measured by Trevitt et al. (2010) and the rate coefficient used by McGuire et al. (2018) to model the abundance of benzonitrile in TMC-1. Within the experimental uncertainty the measured rate coefficient for the $\mathrm{CN}+\mathrm{C}_{6} \mathrm{H}_{6}$ reaction remains essentially constant between 15 and $295 \mathrm{~K}$. The weighted average of all of the rate coefficients between 15 and $295 \mathrm{~K}$ is $4.4 \pm 0.2 \times 10^{-10} \mathrm{~cm}^{3} \mathrm{~s}^{-1}$.

Care was taken to ensure photodissociation of $\mathrm{C}_{6} \mathrm{H}_{6}$ was negligible during the kinetics experiments as reaction between the phenyl radical and $\mathrm{CN}$ is expected to be rapid. The absorption cross-section for benzene at $248 \mathrm{~nm}$ is $1.4 \times 10^{-19} \mathrm{~cm}^{2}$ (Nakashima \& Yoshihara 1982) yielding a maximum phenyl radical concentration of less than $10^{10} \mathrm{~cm}^{-3}$ at the laser fluence used of $\sim 25 \mathrm{~mJ} \mathrm{~cm}^{-2}$. Kovács et al. (2009) have suggested the possibility of two-photon absorption via the ${ }^{1} \mathrm{~B}_{2 u}$ state, leading to dissociation of benzene; they found a high total absorption cross-section for the second photon of $2.8 \times 10^{-17} \mathrm{~cm}^{2}$. For the highest benzene concentration used here $\left(\sim 1 \times 10^{14} \mathrm{~cm}^{-3}\right)$ and with the laser fluence used, all photolysis of benzene occurs by two-photon dissociation. The resulting phenyl radical concentration is too low $(<1 \%)$ to affect the kinetics of the reaction. The concentrations of the phenyl radical are even lower in reality due to quenching of the excited ${ }^{1} \mathrm{~B}_{2 u}$ state by the buffer gas. To experimentally verify this, we measured the first-order rate coefficient at $T=22.9 \mathrm{~K}$ and $\left[\mathrm{C}_{6} \mathrm{H}_{6}\right]=2 \times 10^{13} \mathrm{~cm}^{-3}$ while varying the excimer laser fluence over the range of $19-30 \mathrm{~mJ} \mathrm{~cm}^{-2}$ and found that $k_{1 \mathrm{st}}$ remained constant.

\section{Discussion}

\subsection{Chemical Kinetics}

The rate coefficients for the reaction of $\mathrm{CN}$ with $\mathrm{C}_{6} \mathrm{H}_{6}$, measured here between 15 and $295 \mathrm{~K}$, are consistent with those previously measured by Trevitt et al. (2010) at 295, 165, and $105 \mathrm{~K}$ with a pulsed CRESU apparatus and PLP-LIF. Trevitt et al. (2010) predicted the reaction would remain rapid at even lower temperatures, as confirmed by our measurements. The lack of temperature dependence observed in both studies is consistent with a barrierless entrance channel and the formation of an addition complex. It has been shown both experimentally and theoretically that addition-elimination reactions between 

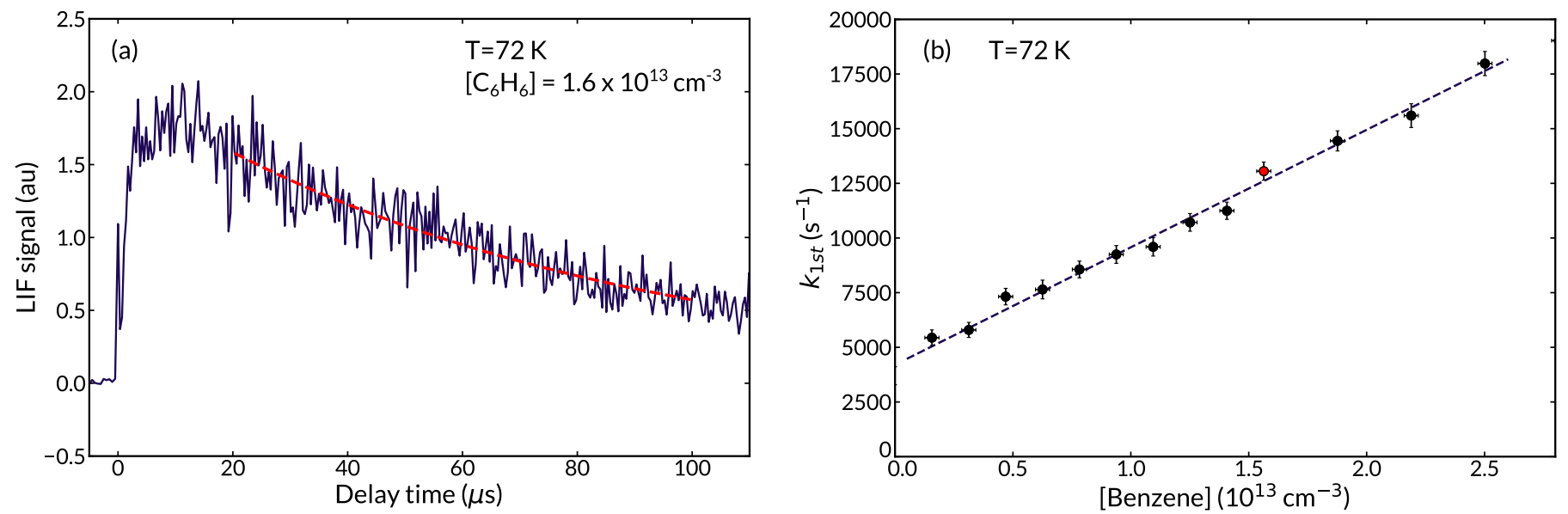

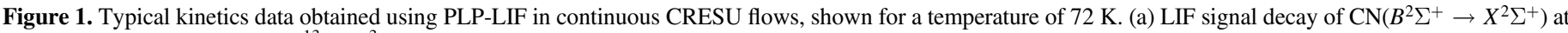

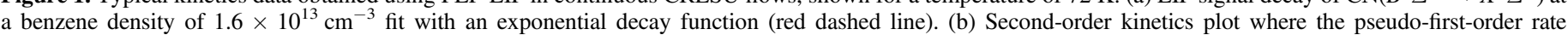

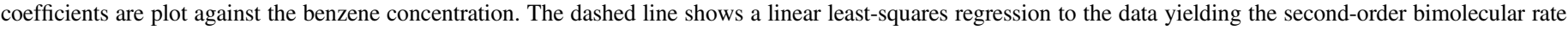
coefficient. The red point corresponds to the $k_{1 \mathrm{st}}$ obtained from (a).

$\mathrm{CN}$ and unsaturated hydrocarbons typically have no barriers in their entrance channel due to the formation of intermediate radical adducts that are relatively stable (Carty et al. 2001). In general, for this class of reactions, there is usually at least one exothermic exit channel to products. Goulay \& Leone (2006) found a similarly large rate coefficient for the reaction between the ethynyl radical $\left(\mathrm{C}_{2} \mathrm{H}\right)$ and benzene with essentially no temperature dependence between 105 and $298 \mathrm{~K}$.

The rate coefficients presented here and those of Trevitt et al. (2010) are similar to those of Woon (2006), who used trajectory calculations to predict the rate coefficients between 50 and $300 \mathrm{~K}$. The calculated rate coefficients ranged from 3.15 and $3.5 \times 10^{-10} \mathrm{~cm}^{3} \mathrm{~s}^{-1}$ when back reactions to reform $\mathrm{CN}+$ $\mathrm{C}_{6} \mathrm{H}_{6}$ are excluded. Woon (2006) also investigated the pressure dependence of the reaction by including back-dissociation of the $\mathrm{C}_{6} \mathrm{H}_{6}-\mathrm{CN}$ intermediate complex using a multiwell treatment and found a slight temperature dependence at $10^{-3}$ mbar, which converges with the high-pressure limit rate coefficient by $50 \mathrm{~K}$. We measured the room temperature rate coefficient at three different pressures for both nitrogen and helium buffer gases and did not observe a pressure dependence. It is important to note, however, that the laboratory experiments were likely conducted within the high-pressure limit.

\subsection{Reaction Products}

Previous theoretical and experimental studies have shown that benzonitrile is the major species produced in the reaction between $\mathrm{CN}$ and benzene, with negligible or no production of the -NC isomer phenylisocyanide nor the H-abstraction product, the phenyl radical. Woon (2006) calculated the reaction potential energy surface and relative product yields and found that while the $\mathrm{C}_{6} \mathrm{H}_{5} \mathrm{CN}+\mathrm{H}$ products form exothermically and barrierlessly, there is a $25 \mathrm{~kJ} \mathrm{~mol}^{-1}$ barrier to form the isocyano product pair $\mathrm{C}_{6} \mathrm{H}_{5} \mathrm{NC}+\mathrm{H}$ after initial barrierless formation of the $\mathrm{C}_{6} \mathrm{H}_{5} \mathrm{NC}$ adduct, which was found to be extremely rare in multiwell calculations.

Crossed-beam experiments conducted under single-collision conditions at much higher energies have demonstrated that the benzonitrile is the main reaction product (Balucani et al. 1999, 2000a, 2000b). Balucani et al. (1999) conducted crossedbeam experiments at collision energies between 19.5 and
$34.4 \mathrm{~kJ} \mathrm{~mol}^{-1}$ as well as electronic structure and RRKM calculations. Neither the $\mathrm{C}_{6} \mathrm{H}_{6} \mathrm{CN}$ adduct nor the phenylisocyanide isomer $\mathrm{C}_{6} \mathrm{H}_{5} \mathrm{NC}$ were found to contribute to the crossed-beam scattering signal. It was concluded that the dominant reaction entrance channel is barrierless leading to the formation of a $\mathrm{CN}$-addition complex that subsequently dissociates to form benzonitrile $+\mathrm{H}$, with the $\mathrm{C}_{6} \mathrm{H}_{5} \mathrm{NC}$ product channel contributing less than $2 \%$.

Lee et al. (2019) studied the reaction of benzene $+\mathrm{CN}$ indirectly using microwave discharge experiments and cavity Fourier-transform microwave spectroscopy, in combination with electronic structure calculations. They found that the reaction produces benzonitrile in high yield, with $<0.1 \%$ relative abundance of phenylisocyanide. Isotopic measurements confirmed that the $\mathrm{CN}$ bond remains intact during the product formation. In contrast to previous computations, they found formation of the iso-adduct requires surmounting a barrier of $\sim 17 \mathrm{~kJ} \mathrm{~mol}^{-1}$ as well as a second barrier for $\mathrm{H}$ atom loss; implying that phenylisocynaide formation should be highly disfavored under low-temperature conditions. While these very sensitive experiments provide important insights into possible reaction pathways, it is important to note that they were not conducted under conditions of kinetic isolation, nor at thermal equilibrium. Under these conditions it is difficult to probe any specific reaction mechanism as multiple reaction paths may contribute and it is unclear how well the productbranching ratios obtained reflect those that would be obtained for the elementary reaction $\mathrm{CN}+\mathrm{C}_{6} \mathrm{H}_{6}$ at a well-defined temperature. Low-temperature kinetics experiments are still needed to identify and confirm the product channel-specific rate coefficients that are critical parameters for models of astrochemical environments and planetary atmospheres.

Chirped-pulse Fourier-transform microwave spectroscopy has recently been combined with CRESU flows to measure the channel-specific rate coefficients at low temperature (Abeysekera et al. 2015). This technique (named Chirped-Pulse in Uniform flows, or CPUF) can be used to establish productbranching ratios at low temperatures, which are critical for astrochemical models but are challenging to obtain experimentally. $K_{a}$-band $(\sim 28-40 \mathrm{GHz})$ and $E$-band $(\sim 60-90 \mathrm{GHz})$ spectrometers have been coupled to the continuous CRESU flows in Rennes that will allow the formation of benzonitrile in 


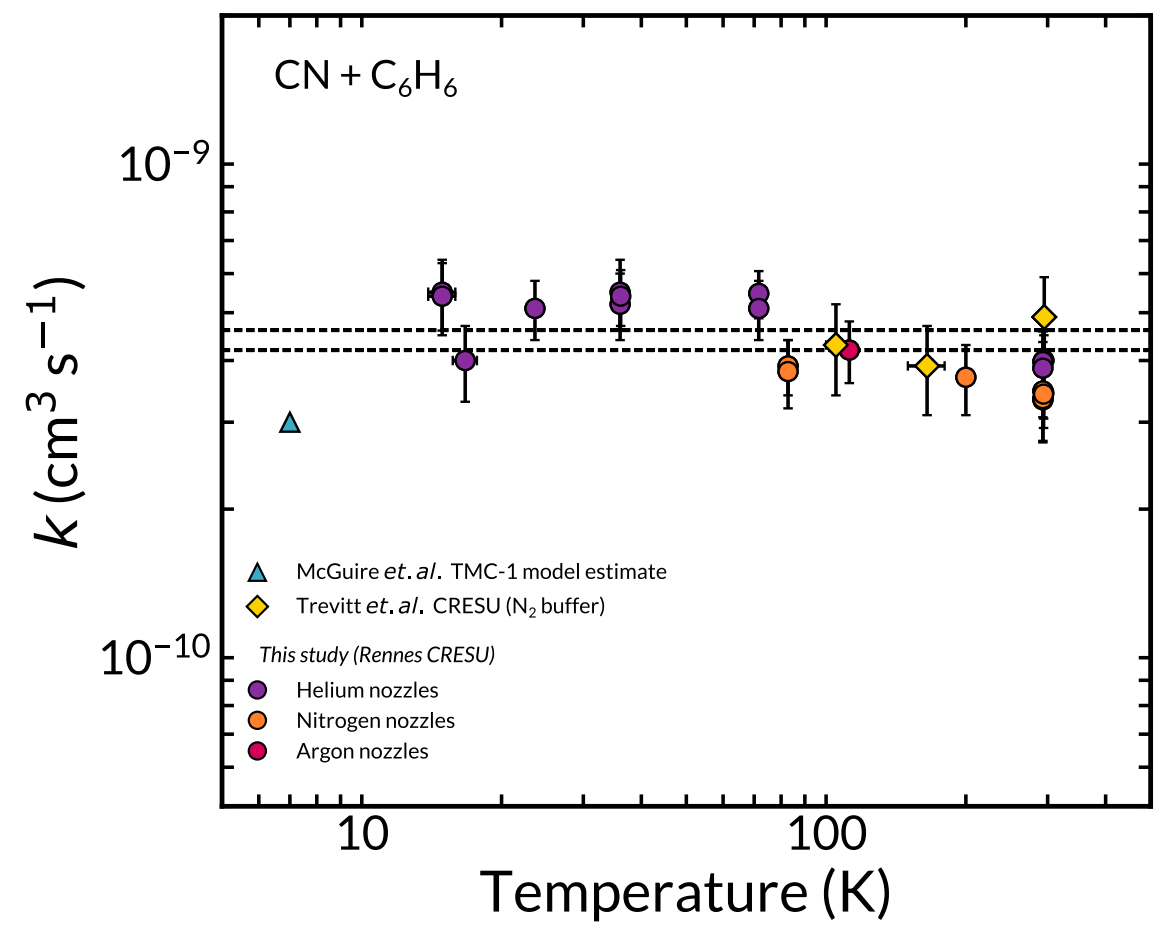

Figure 2. Rate coefficients for the reaction of the $\mathrm{CN}$ radical with benzene as a function of temperature displayed on a log-log scale. The circles are the data taken here using the continuous CRESU in Rennes, with helium buffer gas in purple, nitrogen in orange, and argon in pink. The yellow diamonds are the data taken by Trevitt et al. (2010) using a pulsed CRESU. The teal triangle shows the estimated rate coefficient used by McGuire et al. (2018) to model the abundance of benzonitrile in TMC-1 at $7 \mathrm{~K}$. The dashed lines show the upper and lower limits set by the standard error $( \pm 1 \sigma)$ on the variance weighted mean of the readings at all temperatures.

the $\mathrm{CN}+\mathrm{C}_{6} \mathrm{H}_{6}$ reaction to be measured down to low temperatures. Figure 3 shows how the CRESU flow acts as an efficient rotational refrigerator, shifting the Boltzmann distribution of the rotational lines into the range of the chirpedpulse spectrometers in Rennes. While benzonitrile is expected to be the sole product of $\mathrm{CN}+\mathrm{C}_{6} \mathrm{H}_{6}$, the CPUF technique can be used to measure the product-branching ratios for other multichannel reactions involving $\mathrm{CN}$ and aromatics.

\subsection{Astrophysical Implications}

The rate coefficients measured here for the $\mathrm{CN}$ reaction with benzene are consistent with (albeit somewhat higher than) the value used in the astrochemical model of McGuire et al. (2018) to predict the abundance of benzonitrile in TMC-1, $k(T)=3 \times 10^{-10} \mathrm{~cm}^{3} \mathrm{~s}^{-1}$ (C. N. Shingledecker 2019, private communication). It is therefore unlikely that this reaction is the cause of the discrepancy between the abundance of benzonitrile in their model versus that observed in TMC-1. The large rate coefficients observed over the full temperature range suggest that the reaction should be rapid in astronomical sources that have sufficient density of benzene and $\mathrm{CN}$. Instead, the discrepancy between the observed and modeled abundances is likely due to the underproduction of benzene, due to missing production routes and/or underestimation of the rates for those already in the reaction network.

A photochemical model has been recently developed by Loison et al. (2019) to investigate the production of aromatics in the atmosphere of Titan. While the model predicted significant formation of toluene and ethylbenzene, benzonitrile was not predicted to be abundant due to efficient consumption of $\mathrm{CN}$ by methane.

It is likely that other radicals can add to benzene with similarly rapid rate coefficients, indicating that other benzene

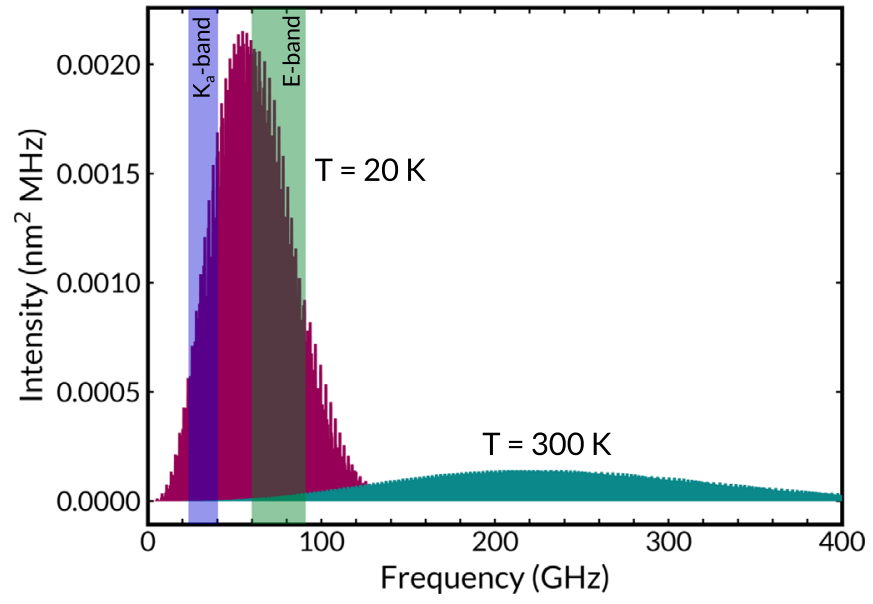

Figure 3. Simulated benzonitrile spectra at $20 \mathrm{~K}$ (magenta) and room temperature (teal). The vertical blocks show the bandwidth of the two CPUF spectrometers in Rennes, one covering the $K a$ band from 26.5 to $40 \mathrm{GHz}$ and the other the $E$ band from 60 to $90 \mathrm{GHz}$. The spectra were simulated using data from the Cologne Database for Molecular Spectroscopy and Pickett's spfit/ spcat program (Pickett 1991).

derivatives may be present in the cold ISM. The reaction of $\mathrm{CH}$ with benzene was measured by Hamon et al. (2000) at $25 \mathrm{~K}$ and found to be similarly rapid with a rate coefficient of $2.7 \times 10^{-10} \mathrm{~cm}^{3} \mathrm{~s}^{-1}$. Rate coefficients for the reaction of $\mathrm{OH}$ with benzene down to $58 \mathrm{~K}$ were presented in a review by Hansmann \& Abel (2007) and were found to be an order of magnitude lower; however, a complete kinetics study has yet to be published. The reaction of $\mathrm{CN}$ with other aromatics may also be rapid and produce products with high dipole moments that could lend themselves to astronomical detection. An alternative route to benzonitrile formation could involve the 
radiative association reaction between the phenyl $\left(\mathrm{C}_{6} \mathrm{H}_{5}\right)$ and $\mathrm{CN}$ radicals, which may be rapid under ISM conditions but has not yet been investigated. The reactions of $\mathrm{CH}$ and $\mathrm{OH}$ with anthracene have both been measured in CRESU flows (Goulay et al. 2005, 2006), though reactions of $\mathrm{CN}$ with aromatics larger than benzene have not yet been studied.

The formation of benzene under interstellar conditions is not as well understood and is a major source of uncertainty in astrochemical models of aromatic chemistry. Jones et al. (2011) investigated benzene formation via the reaction of $\mathrm{C}_{2} \mathrm{H}$ with 1,3-butadiene. Benzene was observed at significant fractions $(30 \% \pm 10 \%)$ with the dominant reaction product the thermodynamically less stable isomer, hexa-1,3-dien-5-yne. However, Lockyear et al. (2015) also studied the products of this reaction using synchrotron photoionization mass spectrometry. The photoionization spectra indicated that fulvene is the major reaction product, with a branching fraction of $\sim 60 \%$. They did not detect benzene as a product and placed an upper limit on the production of benzene and hexa-1,3-dien-5-yne isomers of $45 \%$. Lee et al. (2019) likewise observed evidence for fulvene formation in a microwave discharge containing $\mathrm{HC}_{3} \mathrm{~N}\left(\mathrm{a} \mathrm{C}_{2} \mathrm{H}\right.$ precursor) and 1,3-butadiene. The discrepancy may be due the high collisional energy of the crossed-beam experiment, emphasizing the importance of studying reactions under both single-collision and thermal conditions. The main formation route for benzene in the kida.uva.2014 network is the dissociative recombination of $\mathrm{C}_{6} \mathrm{H}_{7}^{+}$with an electron. McGuire et al. (2018) also added the reaction between $\mathrm{C}_{2} \mathrm{H}+1,3$-butadiene to the kida.uva.2014 network with a rate coefficient of $3 \times 10^{-10} \mathrm{~cm}^{3} \mathrm{~s}^{-1}$. It is therefore possible that the benzene abundance in their model is overestimated since they do not account for the low (or zero) fraction of benzene likely produced by this reaction at low temperatures. In addition, 1,3-butadiene has not been detected in the ISM and thus it is unknown whether the reaction could produce significant quantities of benzene in TMC-1.

\section{Conclusions}

The CRESU technique, combined with PLP-LIF, has been used to measure the kinetics of the reaction between $\mathrm{CN}$ and benzene over the temperature range $15-295 \mathrm{~K}$. We find that the rate coefficients for this reaction do not display an obvious temperature dependence over this temperature range, confirming that the $\mathrm{CN}+\mathrm{C}_{6} \mathrm{H}_{6}$ reaction will remain rapid at temperatures relevant to the cold ISM. These results suggest that benzonitrile is indeed a robust chemical proxy that can be used to infer the abundance of benzene from observations made using radio astronomy. They also indicate that the discrepancy between the observed and modeled abundances of benzonitrile in TMC-1 is likely due to missing or inaccurate kinetic data for benzene production routes.

The authors thank Jonathan Courbe, Jonathan Thiévin, Didier Biet, Ewen Gallou, and Alexandre Dapp for technical support. The authors thank Brett McGuire, Christopher Shingledecker, and Mitchio Okumura for helpful discussions. J.P.M. was supported by the National Science Foundation Graduate Research Fellowship (NSF GRFP) and the National Science Foundation Graduate Research Opportunities Worldwide (NSF GROW) programs. J.P.M. would also like to thank the Office for Science and Technology of the Embassy of France in the United States for a Chateaubriand Fellowship.
The authors acknowledge funding from the European Research Council (ERC) under the European Union's Horizon 2020 research and innovation programme under grant agreement 695724-CRESUCHIRP and under the Marie SkłodowskaCurie grant agreement 845165 -MIRAGE. The authors are also grateful for support from the European Regional Development Fund, the Region of Brittany and Rennes Metropole. This work was supported by the French National Programme "Physique et Chimie du Milieu Interstellaire" (PCMI) of CNRS/INSU with INC/INP co-funded by CEA and CNES.

Software: SciPy (Virtanen et al. 2019), NumPy (van der Walt et al. 2011), Matplotlib (Hunter 2007), StatsModels (http://www.statsmodels.org).

\section{ORCID iDs}

Ilsa R. Cooke (1) https://orcid.org/0000-0002-0850-7426 Divita Gupta (ㄷ) https://orcid.org/0000-0002-6639-4909 Joseph P. Messinger (1) https://orcid.org/0000-00017305-3945

Ian R. Sims (1) https://orcid.org/0000-0001-7870-1585

\section{References}

Abeysekera, C., Joalland, B., Ariyasingha, N., et al. 2015, J. Phys. Chem. Lett., 6,1599

Balucani, N., Asvany, O., Chang, A. H. H., et al. 1999, JChPh, 111, 7457

Balucani, N., Asvany, O., Huang, L. C. L., et al. 2000a, ApJ, 545, 892

Balucani, N., Asvany, O., Osamura, Y., et al. 2000b, P\&SS, 48, 447

Carty, D., Le Page, V., Sims, I. R., \& Smith, I. W. M. 2001, CPL, 344, 310

Cernicharo, J., Heras, A. M., Tielens, A. G. G. M., et al. 2001, ApJL, 546, L123

Cherchneff, I., Barker, J. R., \& Tielens, A. G. G. M. 1992, ApJ, 401, 269

Chiar, J. E., Tielens, A. G. G. M., Adamson, A. J., \& Ricca, A. 2013, ApJ, 770,78

Cooke, I. R., \& Sims, I. R. 2019, ESC, 3, 1109

Dwek, E., Arendt, R. G., Fixsen, D. J., et al. 1997, ApJ, 475, 565

Felps, W. S., Rupnik, K., \& McGlynn, S. P. 1991, JPhCh, 95, 639

Goulay, F., \& Leone, S. R. 2006, JPCA, 110, 1875

Goulay, F., Rebrion-Rowe, C., Biennier, L., et al. 2006, JPCA, 110, 3132

Goulay, F., Rebrion-Rowe, C., Le Garrec, J. L., et al. 2005, JChPh, 122, 104308

Gupta, D., Cheikh Sid Ely, S., Cooke, I. R., et al. 2019, JPCA, 123, 9995

Hamon, S., Le Picard, S. D., Canosa, A., Rowe, B. R., \& Smith, I. W. M. 2000, JChPh, 112, 4506

Hansmann, B., \& Abel, B. 2007, Chem. Phys. Chem, 8, 343

Hunter, J. D. 2007, CSE, 9, 90

James, P. L., Sims, I. R., Smith, I. W. M., Alexander, M. H., \& Yang, M. 1998, JChPh, 109, 3882

Jones, B. M., Zhang, F., Kaiser, R. I., et al. 2011, PNAS, 108, 452

Kaiser, R. I., Parker, D. S. N., \& Mebel, A. M. 2015, ARPC, 66, 43

Kovács, T., Blitz, M. A., Seakins, P. W., \& Pilling, M. J. 2009, JChPh, 131, 204304

Kraemer, K. E., Sloan, G. C., Bernard-Salas, J., et al. 2006, ApJL, 652, L25

Lee, K. L. K., McGuire, B. A., \& McCarthy, M. C. 2019, PCCP, 21, 2946

Lockyear, J. F., Fournier, M., Sims, I. R., et al. 2015, IJMSp, 378, 232

Loison, J. C., Dobrijevic, M., \& Hickson, K. M. 2019, Icar, 329, 55

Lovas, F. J., McMahon, R. J., Grabow, J.-U., et al. 2005, JACS, 127, 4345

Malek, S. E., Cami, J., \& Bernard-Salas, J. 2011, ApJ, 744, 16

McGuire, B. A., Burkhardt, A. M., Kalenskii, S., et al. 2018, Sci, 359, 202

Nakashima, N., \& Yoshihara, K. 1982, JChPh, 77, 6040

O’Halloran, M. A., Joswig, H., \& Zare, R. N. 1987, JChPh, 87, 303

Pickett, H. M. 1991, JMoSp, 148, 371

Seabold, S., Perktold, J., Fulton, C., et al. 2017, statsmodels/statsmodels, Version 0.8.0, Zenodo, doi:10.5281/zenodo.275519

Sims, I. R., Queffelec, J., Defrance, A., et al. 1994, JChPh, 100, 4229

Tielens, A. G. G. M., \& Charnley, S. B. 1997, in Planetary and Interstellar Processes Relevant to the Origins of Life, ed. D. C. B. Whittet (Berlin: Springer), 23

Trevitt, A. J., Goulay, F., Taatjes, C. A., Osborn, D. L., \& Leone, S. R. 2010, JPCA, 114, 1749

van der Walt, S., Colbert, S. C., \& Varoquaux, G. 2011, CSE, 13, 22 
Virtanen, P., Gommers, R., Oliphant, T. E., et al. 2019, CoRR, arXiv:1907. 10121

Wakelam, V., Loison, J.-C., Herbst, E., et al. 2015, ApJS, 217, 20
Woods, P. M., Millar, T. J., Zijlstra, A. A., \& Herbst, E. 2002, ApJL, 574, L167

Woon, D. E. 2006, CP, 331, 67 